\title{
Colloidal liquid crystals in square confinement: isotropic, nematic and smectic phases
}

\author{
Louis B.G. Cortes, Yongxiang Gao, Roel P.A. Dullens and \\ Dirk G.A.L. Aarts \\ ${ }^{1}$ Department of Chemistry, Physical and Theoretical Chemistry Laboratory, \\ University of Oxford, South Parks Road, Oxford OX1 3QZ, UK \\ E-mail: louis.cortes@chem.ox.ac.uk
}

\begin{abstract}
We report on the confinement of colloidal liquid crystals in three dimensional chambers with a square footprint. To this end we use colloidal silica rods and exploit their relatively large density difference with respect to the dispersing solvent to study isotropic, nematic and smectic phases confined into a single chamber. Combining laser scanning confocal microscopy and soft-lithography techniques enables us to characterize the configurations down to the single particle level. We will focus on the smectic phase and compare to recent theories and simulations.
\end{abstract}

Keywords: Lyotropic Liquid Crystal, Isotropic, Nematic, Smectic, Domains, Brownian Motion, colloids

Submitted to: J. Phys.: Condens. Matter

\section{Introduction}

Recent years have witnessed an increasing interest in the confinement of colloidal liquid crystals, in theory $[1,2,3,4,5,6,7,8,9,10,11,12,13]$, simulations $[2,14,15,16,17,18,19,20]$, and experiments $[10,13,20]$. The interest arises from the interplay between "simple" packing problems satisfying local boundary conditions, competing elasticities and defects. Advances in lithography techniques mean that confining length-scales for molecular Liquid Crystals (LCs) become of the order of the extrapolation length $\xi[21,22,23]$, which is set by the ratio between the elasticity $K$ and the anchoring strength $W$; for colloidal LCs the confinement may even reach the particle length $L$. Further advances in colloidal model systems, imaging techniques, and image analysis explain why experiments are now catching up with predictions made by theory and simulations, which have benefited from a steady increase in computational power. Finally, the clear connections with confinement of elongated particles in biology, e.g. actin filaments or virus particles in an infected cell [24], give the confinement of colloidal liquid crystals further relevance. 
The focus, however, has largely been on the confinement of the nematic phase, certainly from an experimental point of view. This may be driven by the use of nematics in display technologies. In this paper we will focus our attention on the colloidal smectic phase, here illustrated by confinement in 3D chambers with a square footprint. The goal of the paper is two-fold: (i) to illustrate a model experimental set-up where we may study isotropic, nematic and smectic phases at coexistence in a single confining chamber, down to the single particle level; and (ii) to experimentally demonstrate the richness of the smectic structures confined in squares and to compare to recent theories and simulations.

In particular, to satisfy (i) we make use of a model silica rods system, recently developed by Kuijk et al. [25]. Here we exploit the relatively large mass density of silica to let gravity both induce and confine smectic, nematic, and isotropic phases. The gravitational length of our silica particles, $L_{g}=k_{B} T / m^{\star} g$, with $k_{B}$ the Boltzmann constant, $T$ the temperature, $m^{\star}$ the silica rod's buoyant mass and $g$ earth's acceleration, is of the order of the rod length. Consequently, the confining length scale, the rod length and the gravitational length all are of similar magnitude. This provides a novel way to study confinement. We note that the effect of gravity on LC phase behaviour has been studied in bulk, in a number of theoretical and computational papers, see for example $[26,27,28]$, and in particular [28], which examined a similar ratio between rod diameter and gravitational length as in our work.

In the next section we will give a brief overview of the theoretical background and highlight some of the possible structures that may be observed in confinement (section 2). This will be followed by the experimental section, where both the system and techniques will be introduced (section 3). Results will be presented and discussed in section 4 . We will conclude in section 5.

\section{Theoretical Background}

For a system of hard rods the competition between orientational and translational entropy leads to the formation of LCs phases, ranging from isotropic liquid (I) at low densities, to nematic $(\mathrm{N})$, smectic $(\mathrm{Sm})$ and/or columnar $(\mathrm{Col})$ and finally crystalline $(\mathrm{Cr})$ phases as a function of density [29]. For a recent review on the role of particle shape, polydispersity, and aspect ratio for particles with hard interactions, see [30]. Confining any of these phases will increase the possible structures: here we focus on the confinement of hard rods where the walls have degenerate planar anchoring conditions, in the strong anchoring limit. Homeotropic anchoring conditions would increase the complexity of the phase space, but we are unaware of such anchoring conditions for colloidal rods. We will start with the isotropic phase, followed by the nematic and finally the smectic phase. Moreover, we will consider the confinement as quasi-2D, where for the time being the third dimension is largely ignored.

Isotropic phase Approaching the I-N transition a confined isotropic phase may start

to show ordering near the walls. Such surface induced ordering has been predicted in 


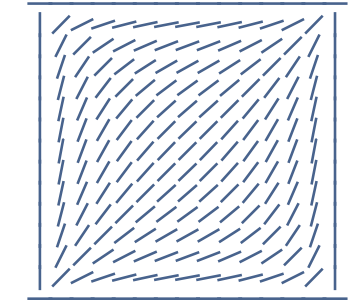

(a)

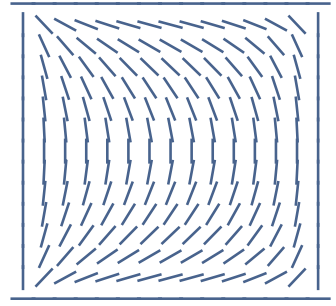

(b)



(c)

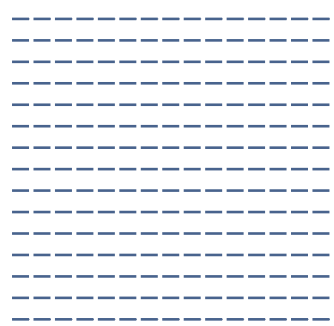

(d)

Figure 1. Local average orientation of lyotropic LCs in square confinement. Figures (a) and (b) display the equilibrium nematic orientation for diagonal and bend configurations. Figures (c) and (d) are sketches of a smectic bridge configuration and of a uniform smectic state.

theory and simulations $[2,3,15]$, but not yet observed experimentally. Even closer to the I-N transition the system may show capillary nematization $[2,3,15,4,6,8]$, i.e. the nematic phase becomes stable below the bulk IN transition concentration. This has only very recently been observed experimentally for a colloidal LC [31]. Generally, theoretical predictions for capillary nematization have been made for a system confined between two parallel hard walls. If one considers the phenomenon for a square chamber the boundary conditions will lead to a shift of the capillary nematization transition. Here wo do not consider this further, but instead briefly discuss the nematic phase from within the bulk nematic region.

Nematic phase. The interplay between boundary conditions and elastic deformations leads to a host of possible structures. These can to a large extent be qualitatively captured within the Oseen-Frank framework. Taking the splay and bend elasticities (characterized by the elastic constants $K_{1}$ and $K_{3}$ ) into account, assuming Dirichlet boundary conditions and minimizing the elastic free energy, leads to a set of Euler-Langrange equations, see [13] for recent work on this. Within the one constant approximation $\left(K_{1}=K_{3}\right)$ minimizing the elastic free energy amounts to solving the Laplace equation for the orientation of the director. Recent work [13] has shown that the predicted structures are in good agreement with a colloidal model system, were both semi-flexible wild type $f d$ virus (with $K_{1} \approx K_{3}$ ) and the stiff mutant virus Y21M (with $K_{1}>K_{3}$ ) were studied. Some of the predicted structures are reproduced here by numerically solving the Laplace equation using Mathematica, see figure 1 (a) and (b). The diagonal state (a), with opposing corners of the same defect strength \pm 1 , was found to be of lower energy with respect to the bend state (b), where opposing corners have opposite strength.

Smectic phase Confinement of the smectic phase has received considerably less attention. A recent theoretical study using the restricted orientation approximation (Zwanzig model) explored the behaviour of 2D hard rectangular particles with aspect ratios ranging from 1 to 6 and confinement between 5 and 10L [11]. Notably, a "bridge" smectic configuration was found, which is characterized by 3 smectic domains, see the 
sketch in figure 1 (c). Two small domains assure planar anchoring on two opposite sides, and a large domain connects the two other sides. In contrast, a recent $\mathrm{MC}$ simulation of hard spherocylinders with aspect ratios 10-38 in quasi-2D confinement with the length of the wall $W \approx 3 L$ shows a single smectic domain, although the focus of that paper was on the nematic phase. Another very recent MC study of hard rectangles in 2D with an aspect ratio 20 and $\mathrm{W}=3-10 \mathrm{~L}$ did show the bridge smectic, in agreement with the theoretical predictions, although the different domains are less well spatially defined. The authors of [19] raise the question whether the occurrence of the bridge state depends on the detailed particle shape, i.e. rectangular vs. spherocylindrical. In our experimental system, however, one end of the rod has a spherocylindrical cap, whereas the other end has a flat cap.

We will compare our experimental observations in light of the results from theory and simulations after the experimental method section.

\section{Method}

\subsection{Synthesis}

We followed the method developed by [25] to synthesize non-fluorescent silica rods with an aspect ratio $L / D>10$, with $L$ and $D$ particle length and diameter. This is an excellent models system for the phase behaviour of hard rods [32]. In a two liter container full dissolution of 120g of polyvinylpyrrolidone (PVP40 Sigma-Aldrich) into 1.2L of 1-pentanol (138975 Sigma-Aldrich) was obtained after $3 \mathrm{~h}$ in a sonication bath. As a next step, $32.4 \mathrm{~mL}$ of deionized water (Milli-Q), $7.2 \mathrm{~mL}$ of sodium citrate $0.18 \mathrm{M}$ in water (S4641 Sigma-Aldrich) and $128 \mathrm{~mL}$ of ethanol (32221 Sigma-Aldrich) were added to the solution. This one was then shaken in a steady circular motion for 100s. A final addition of $24.3 \mathrm{~mL}$ of ammonium hydroxide solution (09861 Fluka Sigma-Aldrich) and $10.8 \mathrm{~mL}$ of tetraethyleorthosilicate (157811000 Fisher Scientific) was performed. The solution was shaken again for 40s, and left for 4 hours. Following this, $5.4 \mathrm{~mL}$ of tetraethyleorthosilicate were added, the solution was finally shaken for $10 \mathrm{~s}$ and left for $15 \mathrm{~h}$.

Fluorescent rods were also synthesized by using the same protocol with a slightly different recipe. The amount of chemicals was the following: PVP $5 \mathrm{~g}$, pentanol 113g, water $3 \mathrm{~mL}$, sodium citrate $0.6 \mathrm{~mL}$, ethanol $9 \mathrm{~mL}$, ammonia $2.2 \mathrm{~mL}$, TEOS $0.9 \mathrm{~mL}$. In addition, as an exta-step, $834 \mu \mathrm{L}$ of dying agent solution was added, and the mixture shaken for 15s. The dying solution was made up of $5 \mathrm{~mL}$ of ethanol (32221 SigmaAldrich), $35.5 \mathrm{mg}$ of rhodamine b isothiocyanate (283924 Aldrich) and $35 \mu \mathrm{L}$ of (3aminopropyl)triethoxysilane (741442 Aldrich) stirred overnight.

After the synthesis the suspensions were cleaned. As a first cleaning step, the rods were centrifuged for $70 \mathrm{~min}$ at $2400 \mathrm{~g}$. As a second step, the sediments were collected, dispersed into ethanol and centrifuged again at 2000g for $20 \mathrm{~min}$. This second step was repeated 8 times; from the 3 rd to the 5 th time particles were dispersed in water instead 


\begin{tabular}{|c|c|c|c|}
\hline & Ab. & Undyed & Dyed \\
\hline Length & $L$ & $4.9 \pm 1.2 \mu \mathrm{m}$ & $4.3 \pm 0.4 \mu \mathrm{m}$ \\
\hline Diameter & $D$ & $0.39 \pm 0.09 \mu \mathrm{m}$ & $0.38 \pm 0.04 \mu \mathrm{m}$ \\
\hline Aspect ratio & $L / D$ & $12 \pm 3$ & $11 \pm 1$ \\
\hline Gravitational length in water & $L_{g}$ & $0.8 \mu \mathrm{m}$ & $1.0 \mu \mathrm{m}$ \\
\hline
\end{tabular}

Table 1. Silica rods properties. Measurement have been performed on scanning electron microscope images. \pm sign indicate standard deviation from average value over about 200 rods.

of ethanol.

To remove really big rods a centrifugation at $250 \mathrm{~g}$ for 5 minutes was performed, keeping the supernatants. Normal sedimentation was then used to isolate the longer rods, and thus reduce the polydispersity.

The rods used in the experiments are shown in figure 2 ; the rods properties are summarized in table 1.
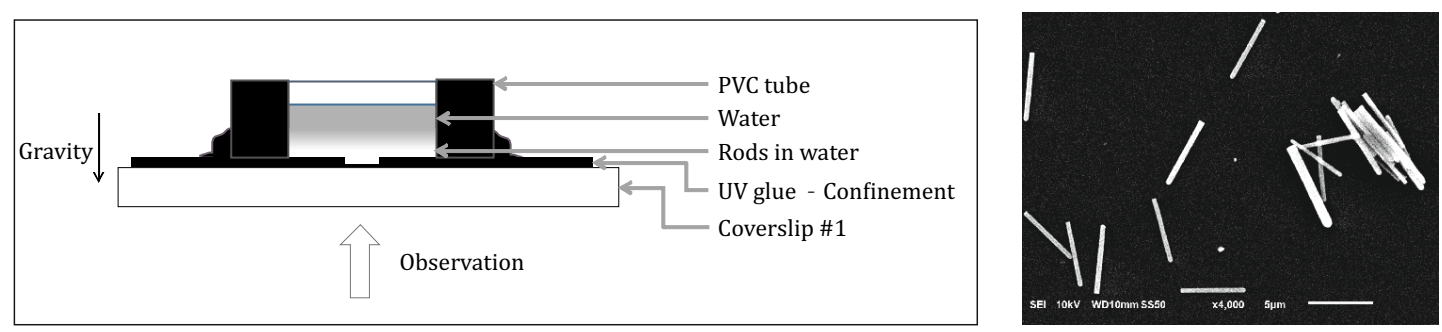

Figure 2. Experimental cell and scanning electron microscopy image of silica rods described in the method section.

\subsection{Techniques}

Experiments were performed with the cell which is schematically shown in figure 2. It is composed of two parts: firstly, a cover-slip on which a layer of glue, containing the micrometric cavities, was covalently bonded; secondly, a PVC tube to form the reservoir.

Standard soft lithography techniques were used to make a PDMS stamp with inverted square cavities. In order to have walls as straight as possible, a chrome mask was used during the polymerization step, rather than a polyester mask. The PDMS stamp was then used to make UV-glue cavities. This last step was realized by dropping NOA81 on a pre-washed cover-slip, and pressing it with the PDMS stamp, taking care to avoid any air bubbles. Before removing the PDMS stamp the glue was polymerized with a UV lamp. The PVC tube was then glued with NOA81. The cell was closed by glueing another cover-slip on the top to prevent evaporation (not shown in figure 2).

For clarity, only one cavity is shown in figure 2. However, the actual size of cavities being smaller than $400 \mu \mathrm{m}$, a large number of cavities can be contained in one cell. Moreover, we note that the final cavity can take any shape imaginable. Furthermore, a 
minimal lateral size of about $5 \mu \mathrm{m}$ can be reached, and the height can range from 1 to at least $100 \mu \mathrm{m}$. Here we use a height of $20 \mu \mathrm{m}$. However, note that the cavities are not directly sealed, but instead were covered by several $\mathrm{mm}$ of water from the reservoir.

In order to fill the sample cell we followed the procedure below. First, the cell was plasma cleaned for $1 \mathrm{~min}$, in order to make the UV-glue hydrophilic, and avoid trapping of air bubbles in the cavities. Then, the cell was filled with water and left open. Rods were then added to the top. Because of their small gravitational length compared to the size of the cell they sedimented in the cavities. Thus, by gradually adding rods to the cell isotropic, nematic and smectic phases can subsequently be formed and stabilized simultaneously in the cavities. We use two different laser scanning confocal microscopes to scan our samples in 3D. One is a Thorlabs system coupled with an olympus microscope and 60x apochromat objective. The other is a Zeiss confocal coupled with a x63 apochromat objective. Because of the refractive index mismatch between water and silica, light scattered by the rods can be collected by a photomultiplier and used to image them. This allowed us to visualize all the rods without the need for dye. Simultaneously, we can visualize and easily track rods labelled with fluorescence by collecting fluorescence light on a second photomultiplier. An alternative approach was followed in [33], which allowed to both visualize and track all rods, but typically leads to heavier rods with a lower aspect ratio, which reduces the nematic region.

\section{Results and discussion}

Figure 3 illustrates the feasibility of our experimental approach. We show typical snapshots for a system with $W \approx 15 L$, and a height $H \approx 4 L$. After filling the sample cell with a dispersion of the colloidal rods, the rods sediment under gravity, fill the chamber and different LC phases emerge.

Starting near the top of the chamber, at a distance from the bottom $z \approx 16 \mu \mathrm{m}$, we observe the isotropic phase characterized by random positions and orientations of the rods, figure 3 (I). Since the rods are dispersed in water the refractive index mismatch means that the images are somewhat noisy. We are detecting the scattered laser light and image the sample through the denser LC phases. This can in principle be overcome by matching the refractive index of the rods and the solvent; however, the isotropic phase is not the focus of the current work.

Going down in the cell the rod density smoothly increases and at a height of $z \approx 11 \mu \mathrm{m}$, we clearly see the hallmarks of the nematic phase, figure $3(\mathrm{~N})$. Rods show orientational order. Matching the planar anchoring conditions leads in this particular case to a deformation, which has defects in all 4 corners and of equal charge and two defects in the central part of the chamber. This particular nematic director seems to match the underlying smectic orientational order, as will be discussed later. The nematic phase turns out to be relatively thin in this particular case, around $6 \mu \mathrm{m}(z=8-14 \mu \mathrm{m})$. We note that Savenko and Dijkstra [28], in their bulk study of hard spherocylinders with comparable $L_{g}$ but lower aspect ratio $L / D=5$, also found a thin nematic layer. 
Going further down in the cell, the nematic-smectic transition occurs at a height $z=6-8 \mu \mathrm{m}$, with a clear smectic phase $(\mathrm{Sm})$ at the bottom of the cell $3(\mathrm{Sm})$. This will be discussed in more detail in the next section. Having the 3 phases at coexistence in a single chamber has the advantage that it becomes possible to take z-stacks by means of the confocal microscope and directly observe the three phases in a single image, see figure 3 (SW); The smectic layers can be clearly observed including the smectic regions where the domains lie perpendicular to the filed of view. The set-up further offers the possibility to see the I-N and N-Sm interfaces, which will be reported in a later study.

Finally, note that adding a tracer amount of fluorescent rods allows imaging for example single particle diffusion in dense phases [34], which we will not explore here.

(I)

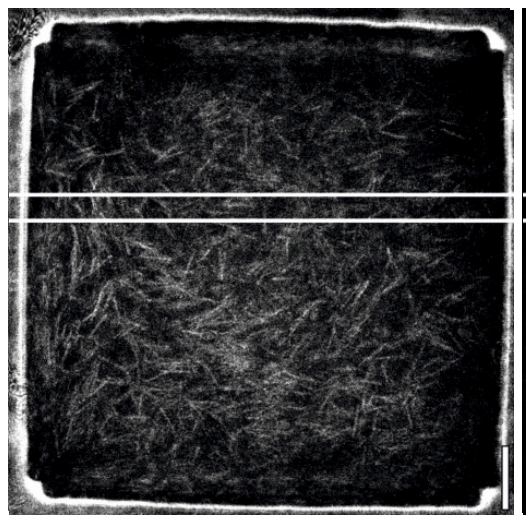

$(\mathrm{N})$

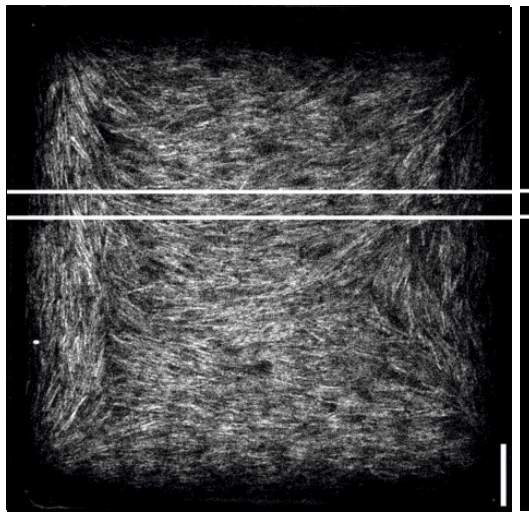

$(\mathrm{Sm})$

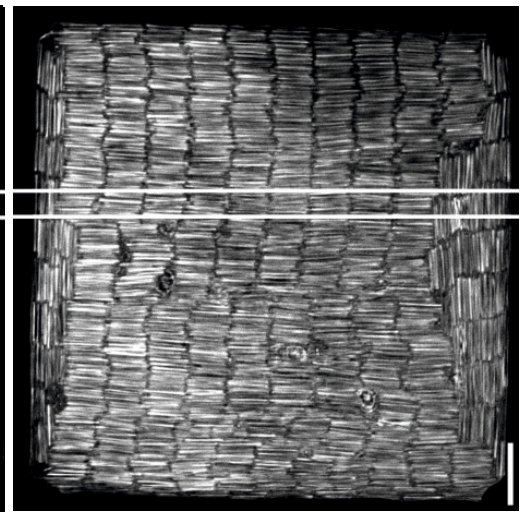

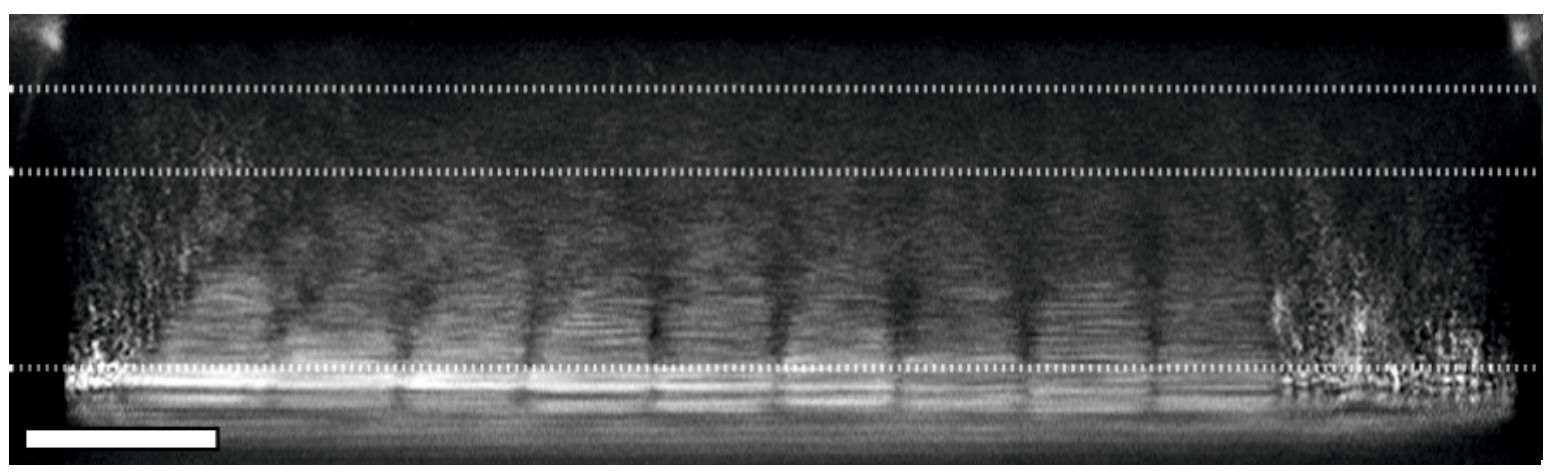

$(\mathrm{SW})$

Figure 3. The top row shows LSCM images (scattered light) of coexisting isotropic (I), nematic $(\mathrm{N})$ and smectic $(\mathrm{Sm})$ phases confined in a $W \approx 15 L$ cavity. The bottom row shows a side view (SW) reconstructed from the location indicated by the double solid lines and for $z$ ranging from $z=-4 \mu \mathrm{m}$ to $z=21 \mu \mathrm{m}$ with respect to the cell bottom. The dotted lines indicate the height where the top row snapshots were taken; from the bottom to the top $(\mathrm{Sm}): z \approx 1 \mu \mathrm{m},(\mathrm{N}): z \approx 11 \mu \mathrm{m},(\mathrm{I}): z \approx 16 \mu \mathrm{m}$. All scale bars denote $10 \mu \mathrm{m}$. See the supplementary movie for a $z$-stack corresponding to the set of images. 


\subsection{Observation of the smectic phase}

Figure 4 shows the smectic phase in square confinement ranging from $W \approx 5 L$ to $W \approx 40 L$, with a constant height $H \approx 4 L$. The left hand panels display LSCM images after a bandpass filter (1-40pixels) has been applied; for the second column we have used built-in Mathematica functions to detect the rods (Functions used were: LocalAdaptativeBinarize and MorphologicalComponents). Not all rods are correctly identified but the method is suitable for our purpose. By colouring the rods depending on their orientation it becomes clear that locally the planar boundary conditions are satisfied. For the smallest squares, up to $W \approx 10 L$, the system chooses to split into 3 smectic domains, with 2 small domains covering the width of two opposite walls, and a large domain bridging the two other square sides. For the square with $W \approx 20 L$, we see a similar behaviour, although a small domain trapped in the large domain can be observed. However, for the larger square $W \approx 40 L$, the structure is different; this will be discussed later.

The typical spacing between the smectic layers can either be measured manually (in real space) or by Fourier transforming the images (except for the two smallest squares). Within the measurement error, the two techniques give similar results. We find for all squares a layer spacing between $6.2 \mu \mathrm{m}<\lambda<7.2 \mu \mathrm{m}$, which corresponds to $1.3<\lambda / L<1.5$. This is a bit larger than theoretical predictions [35, 36]. However, we have underestimated the rod length for the smectic phase: we observe fractionation of the rods with on average longer rods in the smectic phase, particularly at the bottom, where the layer spacing is measured.

A closer inspection of the images shows that the small domains can be as thin as 2 or 3 particles diameters, possibly stabilised by the small roughness of the walls of the confining chambers, but wherever the small domains venture out into the bulk system they typically jump by once or twice the smectic layer spacing. This is indicated by arrows in figure 4. Such a commensurate jump may be expected.

As mentioned above we see the bridge state even for the smallest square $W \approx 5 L$. This may be related to the exact value of the confinement: $W=4.7 L=3.5 \lambda$. This incommensurability may suppress the possibility of a uniform state. Furthermore, the non-sharp edges and especially corners or possibly even the exact shape of the particle may play a role.

Returning to the largest square, we observe that the system still satisfies planar anchoring conditions everywhere in the cell, but away from the corners smectic layers bend from one wall to another, neighbouring wall. Closer to the corners this is no longer possible and the rod orientation changes abruptly by $90^{\circ}$. The bending of layers between adjacent sides of the squares was also observed for a smaller square, but this eventually disappeared. The example in figure 5 shows how the smectic structure in a square of size $W \approx 10 L$ reorganizes as a function of time. We thus expect that such a reorganization will eventually happen for the largest square as well.

Finally, we would like to discuss three more points: firstly, during sedimentation the 
density at the bottom of the cell will first reach the isotropic phase, and then the nematic phase. The structure formed here may influence the subsequent smectic configuration. Secondly, the quasi 2D nature of the set-up may play a role in equilibration. The system is inherently 3D, but the rods are confined by gravity to mainly lie along the bottom glass wall. Only occasionally do we find rods standing up along gravity. Thirdly, coming back to the nematic phase: although the side walls have degenerate planar anchoring conditions, this is not the case for the bottom "wall"; indeed, the nematic phase lies on top of the smectic phase. The N-Sm interface will impose its direction on the nematic phase. This effectively changes the anchoring conditions, a result which so far has not been explored in theory or simulation. We might be observing such an effect here: we find a configuration similar to the $U_{1}^{\star}$-like configuration reported in [13] (figure $3(\mathrm{~N})$ ), matching the underlying smectic orientation (figure $3(\mathrm{Sm})$ ), instead of the predicted diagonal state (figure 1 (a)).

\section{Conclusion}

We have introduced a novel experimental set-up to study colloidal LCs in confinement by exploring the relatively large density of our colloidal model particles. By combining soft lithography and confocal microscopy we have shown that the isotropic, nematic and smectic phases can coexist in a single square cavity. We have highlighted the interplay between the observed configurations of the nematic phase and the underlying smectic phase. We then explored the behaviour of the smectic phase in confining chambers of different dimensions and observed the bridge configuration where one domain bridges the cavity, and two smaller domains exist to satisfy the anchoring conditions. The small domains extend into the large domains set by an integer number of smectic layer spacings. We furthermore observe that the smectic layers can bend to meet anchoring conditions at neighbouring walls. The stability of these states and their time evolution warrants further investigation.

Acknowledgements This project has received funding from the European Union's Horizon 2020 research and innovation programme under the Marie Skłodowska-Curie Grant Agreement No 641839.

\section{References}

[1] Zheng Yu Chen and Shi-Min Cui. Phys. Rev. E, 52:3876, 1995.

[2] Y. Mao, P. Bladon, H. N. W. Lekkerkerker, and M. E. Cates. Molecular Physics, 92:151-159, 1997.

[3] Rene van Roij, Marjolein Dijkstra, and Robert Evans. The Journal of Chemical Physics, 113:7689, 2000.

[4] D. De Las Heras, E. Velasco, and L. Mederos. The Journal of chemical physics, 120:4949, 2004.

[5] D. de las Heras, E. Velasco, and L. Mederos. Phys. Rev. E, 74:011709, 2006.

[6] Hendrik Reich and Matthias Schmidt. Journal of Physics: Condensed Matter, 19:326103, 2007.

[7] David L. Cheung and Matthias Schmidt. 131:214705, 2009.

[8] Alexandr Malijevsk and Szabolcs Varga. Journal of Physics: Condensed Matter, 22:175002, 2010. 
[9] P. Patrcio, J.M. Romero-Enrique, N.M. Silvestre, N.R. Bernardino, and M.M. Telo da Gama. Molecular Physics, 109:1067-1075, 2011.

[10] Oliver J. Dammone, Ioannis Zacharoudiou, Roel P. A. Dullens, Julia M. Yeomans, M. P. Lettinga, and Dirk G. A. L. Aarts. Phys. Rev. Lett., 109:108303, 2012.

[11] Miguel González-Pinto, Yuri Martínez-Ratón, and Enrique Velasco. Phys. Rev. E, 88:032506, 2013.

[12] Jeff Z. Y. Chen. Soft Matter, 9:10921-10930, 2013.

[13] Alexander H. Lewis, Ioana Garlea, Jose Alvarado, Oliver J. Dammone, Peter D. Howell, Apala Majumdar, Bela M. Mulder, M. P. Lettinga, Gijsje H. Koenderink, and Dirk G. A. L. Aarts. Soft Matter, 10:7865-7873, 2014.

[14] Joachim Dzubiella, Matthias Schmidt, and Hartmut Löwen. Phys. Rev. E, 62:5081, 2000.

[15] Marjolein Dijkstra, René van Roij, and Robert Evans. Phys. Rev. E, 63:051703, 2001.

[16] Homin Shin, Mark J. Bowick, and Xiangjun Xing. Phys. Rev. Lett., 101:037802, 2008.

[17] Daniel de las Heras and Enrique Velasco. Soft Matter, 10:1758-1766, 2014.

[18] Ioana C. Gârlea and Bela M. Mulder. Soft Matter, 11:608-614, 2015.

[19] Thomas Geigenfeind, Sebastian Rosenzweig, Matthias Schmidt, and Daniel de Las Heras. The Journal of chemical physics, 142:174701, 2015.

[20] Ioana C Gârlea, Pieter Mulder, José Alvarado, Oliver Dammone, Dirk GAL Aarts, M Pavlik Lettinga, Gijsje H Koenderink, and Bela M Mulder. Nature communications, 7:12112, 2016.

[21] C. Tsakonas, A. J. Davidson, C. V. Brown, and N. J. Mottram. Applied Physics Letters, 90:111913, 2007.

[22] T. Lopez-Leon, V. Koning, K. B. S. Devaiah, V. Vitelli, and A. Fernandez-Nieves. Nature Physics, 7:391-394, 2011.

[23] Mykola Tasinkevych, Michael G. Campbell, and Ivan I. Smalyukh. Proceedings of the National Academy of Sciences, 111:16268-16273, 2014.

[24] Marina Soares e Silva, Jose Alvarado, Jeanette Nguyen, Nefeli Georgoulia, Bela M. Mulder, and Gijsje H. Koenderink. Soft Matter, 7:10631-10641, 2011.

[25] Anke Kuijk, Alfons van Blaaderen, and Arnout Imhof. Journal of the American Chemical Society, 133:2346-2349, 2011.

[26] Thierry Biben, Jean-Pierre Hansen, and Jean-Louis Barrat. The Journal of Chemical Physics, 98:7330, 1993.

[27] Vladimir A. Baulin and Alexei R. Khokhlov. Phys. Rev. E, 60:2973, 1999.

[28] S. V. Savenko and Marjolein Dijkstra. Phys. Rev. E, 70:051401, Nov 2004.

[29] P. G. de Gennes and J. Prost. The physics of liquid crystals, 1993.

[30] Luis Mederos, Enrique Velasco, and Yuri Martnez-Ratn. Journal of Physics: Condensed Matter, 26:463101, 2014.

[31] K. E. Klop, R. P. A. Dullens, M. P. Lettinga, S. A. Egorov, and D. G. A. L. Aarts. submitted, 2016.

[32] Anke Kuijk, Dmytro V. Byelov, Andrei V. Petukhov, Alfons van Blaaderen, and Arnout Imhof. Faraday Discuss., 159:181-199, 2012.

[33] T. H. Besseling, M. Hermes, A. Kuijk, B. de Nijs, T.-S. Deng, M. Dijkstra, A. Imhof, and A. van Blaaderen. Journal of Physics: Condensed Matter, 27:194109, 2015.

[34] M. Paul Lettinga and Eric Grelet. Phys. Rev. Lett., 99:197802, 2007.

[35] Glenn T. Evans. Molecular Physics, 77:969-981, 1992.

[36] Marc Esposito and Glenn T. Evans. Molecular Physics, 83:835-845, 1994. 


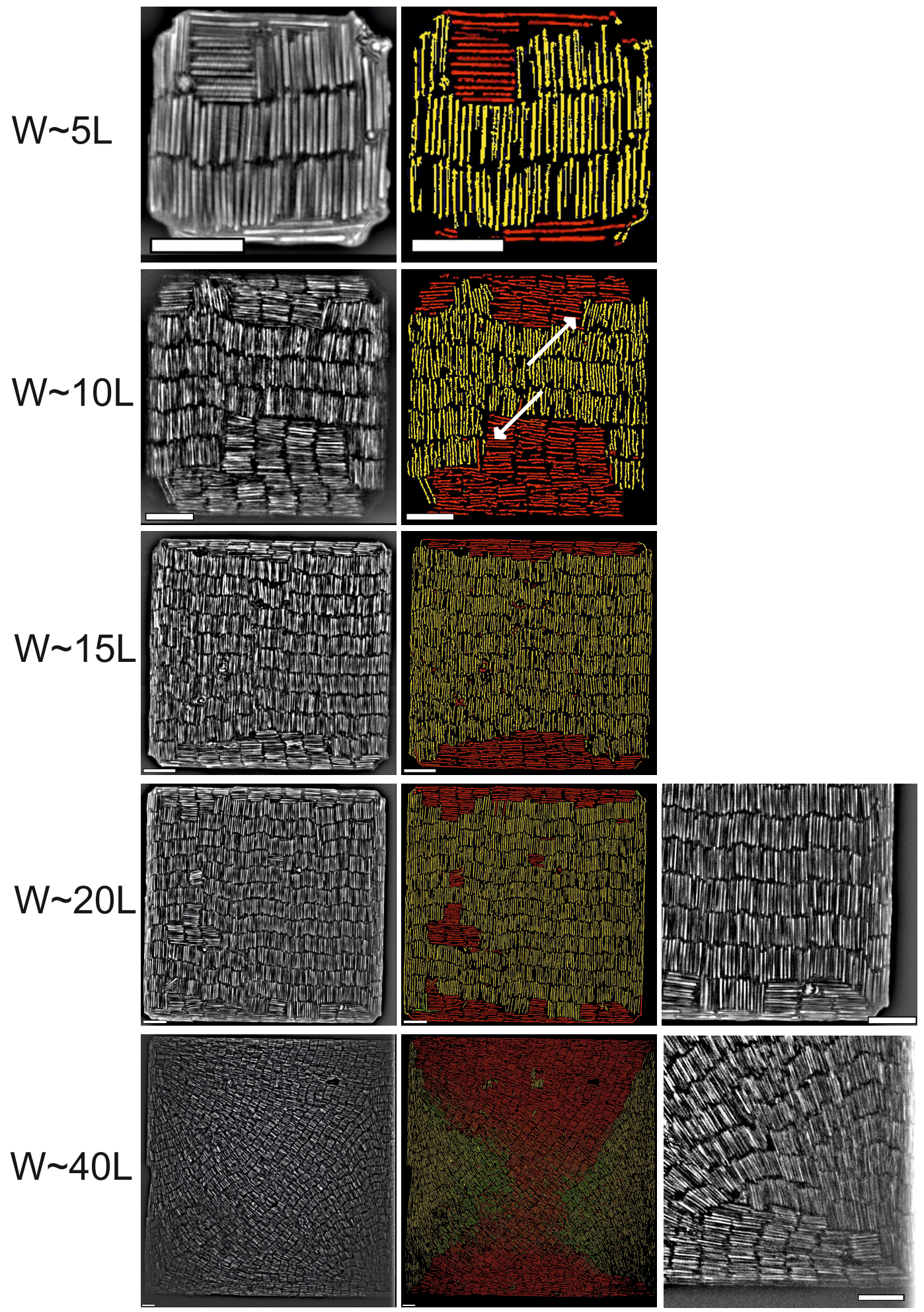

Figure 4. The left hand side column shows bandpass filtered LSCM images (scattered light) of smectic configurations in different square sizes, from top to bottom $W \approx 5 L$, $W \approx 10 L, W \approx 15 L, W \approx 20 L$ and $W \approx 40 L$. The second column shows processed images. Rods are coloured as a function of their orientation. Arrows indicate commensurate jumps in the smectic layers at the domain boundary. The third column shows a zoom in of the bottom right hand side of snapshots of the first column. Scale bars represent $10 \mu \mathrm{m}$. 


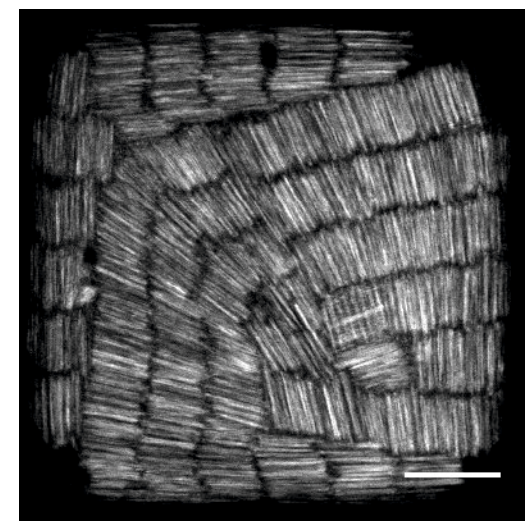

(a)

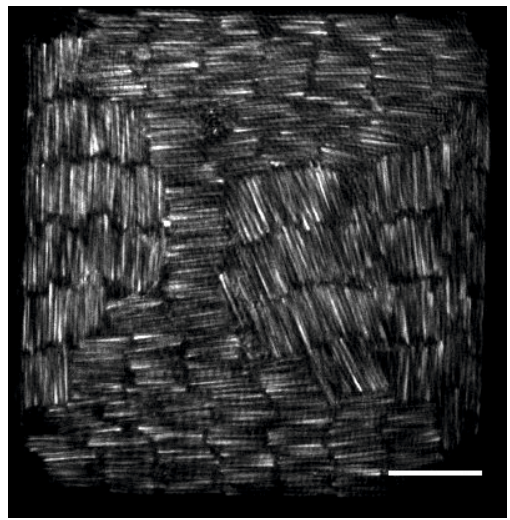

(b)

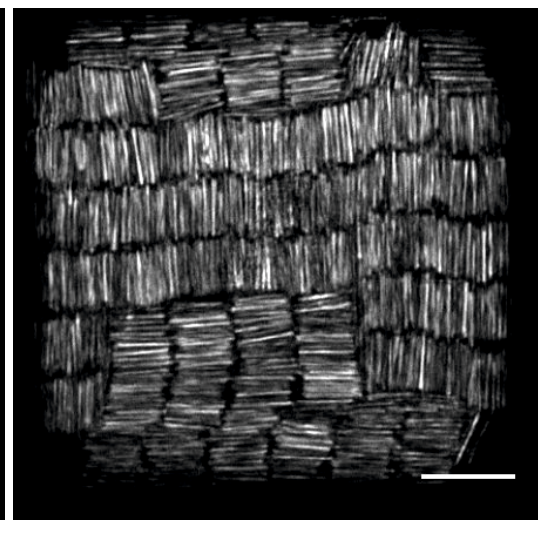

(c)

Figure 5. LSCM images (scattered light) of smectic configurations after filling $t \approx 1 \mathrm{~h}$ (a), at $t \approx 2$ days (b) and at $t \approx 5$ days (c). The square has a side size $W \approx 10 L$. The scale bars represent $10 \mu \mathrm{m}$. 